\title{
Propagation of Uncertainty in Optimal Design of Multilevel Systems: Piston-Ring/Cylinder-Liner Case Study
}

Kuei-Yuan Chan, Michael Kokkolaras, Panos Papalambros and Steven J. Skerlos

University of Michigan

Zissimos Mourelatoes

Oakland University

Reprinted From: Reliability and Robust Design in Automotive Engineering 
All rights reserved. No part of this publication may be reproduced, stored in a retrieval system, or transmitted, in any form or by any means, electronic, mechanical, photocopying, recording, or otherwise, without the prior written permission of SAE.

For permission and licensing requests contact:

SAE Permissions
400 Commonwealth Drive
Warrendale, PA 15096-0001-USA
Email: permissions @ sae.org
Fax: $\quad 724-772-4891$
Tel: $\quad 724-772-4028$

Tel: $\quad 724-772-4028$

For multiple print copies contact:

SAE Customer Service

Tel: $\quad$ 877-606-7323 (inside USA and Canada)

Tel: $\quad$ 724-776-4970 (outside USA)

Fax: $\quad$ 724-776-1615

Email: CustomerService@sae.org

\section{ISBN 0-7680-1319-4}

\section{Copyright $\odot 2004$ SAE International}

Positions and opinions advanced in this paper are those of the author(s) and not necessarily those of SAE. The author is solely responsible for the content of the paper. A process is available by which discussions will be printed with the paper if it is published in SAE Transactions.

Persons wishing to submit papers to be considered for presentation or publication by SAE should send the manuscript or a $\mathbf{3 0 0}$ word abstract of a proposed manuscript to: Secretary, Engineering Meetings Board, SAE.

\section{Printed in USA}




\title{
Propagation of Uncertainty in Optimal Design of Multilevel Systems: Piston-Ring/Cylinder-Liner Case Study
}

\author{
Kuei-Yuan Chan, Michael Kokkolaras, \\ Panos Papalambros and Steven J. Skerlos \\ University of Michigan \\ Zissimos Mourelatoes \\ Oakland University
}

Copyright @ 2004 SAE International

\begin{abstract}
This paper proposes an approach for optimal design of multilevel systems under uncertainty. The approach utilizes the stochastic extension of the analytical target cascading formulation. The reliability of satisfying the probabilistic constraints is computed by means of the most probable point method using the hybrid mean value algorithm. A linearization technique is employed for estimating the propagation of uncertainties throughout the problem hierarchy. The proposed methodology is applied to a piston-ring/cylinder-liner engine subassembly design problem. Specifically, we assess the impact of variations in manufacturing-related properties such as surface roughness on engine attributes such as brake-specific fuel consumption. Results are compared to the ones obtained using Monte Carlo simulation.
\end{abstract}

\section{INTRODUCTION}

Analytical target cascading (ATC) is a methodology for optimal design of hierarchical multilevel systems $[1,2,3]$. Consider a multilevel hierarchical system where outputs of lower-level elements are inputs to higher-level elements (cf. Figure 1), and assume the availability of analysis/simulation models to compute element responses. The ATC objective is to identify element interactions early in the design process, and to determine specifications that yield consistent system design with minimized deviation from design targets. Its usefulness has been demonstrated in several case studies [4, 5, 6, 7]. Global convergence properties of the ATC process have been proven under standard smoothness and convexity assumptions [8]. The ATC process formulates and solves a minimum deviation optimization problem for each element of the multilevel hierarchy. By solving the element optimization problems sequentially in an iterative manner, ATC aims

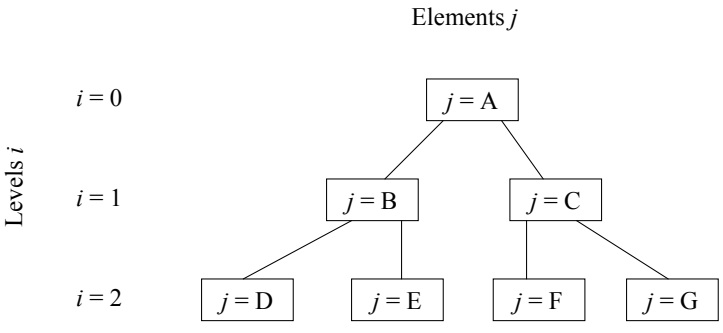

Figure 1: Example of hierarchical multilevel system decomposition

at minimizing discrepancies between the optimal design variable values desired at elements higher in the hierarchy and response values that elements at lower levels can actually deliver. In addition, if design variables are shared among some elements at the same level, their required common final optimal value is coordinated by their parent element at the level above.

The original formulation of ATC does not take into consideration uncertainties that are inherently present in engineering problems. In this paper, we utilize an extension of the ATC formulation to account for uncertainties, and employ a linearization technique to estimate the propagation of the latter throughout the multilevel hierarchy to solve a bi-level problem that considers the optimal design of a piston-ring/cylinder-liner engine subassembly.

The paper is organized as follows. The deterministic formulation of ATC and its stochastic extension are presented in the next section. We then describe the linearization technique used to estimate the propagation of uncertainties in the multilevel hierarchy. The case study is introduced in the following section, and we conclude with a discussion of results and suggestions for future work. 


\section{MATHEMATICAL FORMULATION OF ATC}

Before we proceed with the mathematical formulation of the ATC process, we introduce some notation and make some definitions. Subscripts $i$ and $j$ are used to denote level and element, respectively. For each element $j$ at level $i$, the set $\mathcal{C}_{i j}$ includes the elements that are "children" of this element. For example, in Figure 1 we have $\mathcal{C}_{1 \mathrm{~B}}=\{\mathrm{D}, \mathrm{E}\}$. Responses $\mathbf{r}_{i j}$ are functions of childrenresponses, local design variables, and shared design variables, i.e.,

$$
\mathbf{r}_{i j}=\mathbf{f}_{i j}\left(\mathbf{z}_{i j}\right)=\mathbf{f}_{i j}\left(\mathbf{r}_{(i+1) k_{1}}, \ldots, \mathbf{r}_{(i+1) k_{c_{i j}}}, \mathbf{x}_{i j}, \mathbf{y}_{i j}\right),
$$

where $\mathcal{C}_{i j}=\left\{k_{1}, k_{2}, \cdots, k_{c_{i j}}\right\}$. Shared design variables are restricted to exist only among elements at the same level having the same parent. Tolerance optimization variables $\epsilon^{r}$ and $\epsilon^{y}$ are introduced for coordinating responses and shared variables, respectively. Superscript $u(l)$ is used to denote response and shared variable values that have been obtained at the parent (children) problem(s), and have been cascaded down (passed up) as design targets (consistency parameters). The top-level element of the hierarchy is a special case; the responses cascaded from above are the given system design targets, and since this is the only element of the level, there exist no shared variables.

DETERMINISTIC FORMULATION The mathematical formulation of problem $p_{i j}$ is

$$
\begin{array}{rc}
\min _{\tilde{\mathbf{x}}_{i j}, \epsilon_{i j}^{r}, \epsilon_{i j}^{y}} & \left\|\mathbf{r}_{i j}-\mathbf{r}_{i j}^{u}\right\|_{2}^{2}+\left\|\mathbf{y}_{i j}-\mathbf{y}_{i j}^{u}\right\|_{2}^{2}+\epsilon_{i j}^{r}+\epsilon_{i j}^{y} \\
\text { subject to } & \sum_{k \in \mathcal{C}_{i j}}\left\|\mathbf{r}_{(i+1) k}-\mathbf{r}_{(i+1) k}^{l}\right\|_{2}^{2} \leq \epsilon_{i j}^{r} \\
& \sum_{k \in \mathcal{C}_{i j}}\left\|\mathbf{y}_{(i+1) k}-\mathbf{y}_{(i+1) k}^{l}\right\|_{2}^{2} \leq \epsilon_{i j}^{y} \\
\mathbf{g}_{i j}\left(\mathbf{z}_{i j}\right) \leq \mathbf{0},
\end{array}
$$

where the vector of design variables $\tilde{\mathbf{x}}_{i j}$ consists of vectors $\mathbf{z}_{i j}, \mathbf{y}_{(i+1) k_{1}}, \ldots, \mathbf{y}_{(i+1) k_{c_{i j}}}$, and where $\mathbf{g}_{i j}\left(\mathbf{z}_{i j}\right)$ denote local design inequality constraints. Figure 2 illustrates the information flow of the ATC process at element $j$ in level $i$. Assuming that all the parameters have been updated

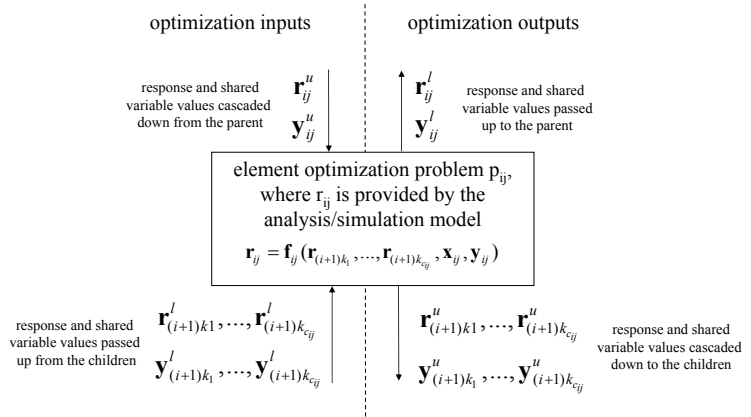

Figure 2: ATC information flow at element $j$ of level $i$

using the solutions obtained at the parent- and childrenproblems, Problem (2) is solved to update the parameters of the parent- and children-problems. This process is repeated until the tolerance optimization variables in all problems cannot be reduced any further.
STOCHASTIC FORMULATION In this section, the ATC formulation is modified to account for uncertainties $[9,10]$. Stochastic quantities are represented by random variables (symbolized by the use of upper case letters), responses are computed as expected values, and constraints are formulated probabilistically. Mathematically, Problem (2) is reformulated as

$$
\begin{array}{cc}
\min _{\tilde{\mathbf{x}}_{i j}, \epsilon_{i j}^{r}, \epsilon_{i j}^{y}} & \left\|\mathbf{E}\left[\mathbf{R}_{i j}\right]-\mathbf{r}_{i j}^{u}\right\|_{2}^{2}+\left\|\mathbf{Y}_{i j}-\mathbf{y}_{i j}^{u}\right\|_{2}^{2}+\epsilon_{i j}^{r}+\epsilon_{i j}^{y} \\
\text { subject to } & P\left[\sum_{k \in \mathcal{C}_{i j}}\left\|\mathbf{R}_{(i+1) k}-\mathbf{r}_{(i+1) k}^{l}\right\|_{2}^{2}>\epsilon_{i j}^{r}\right] \leq P_{f_{1}} \\
& P\left[\sum_{k \in \mathcal{C}_{i j}}\left\|\mathbf{Y}_{(i+1) k}-\mathbf{y}_{(i+1) k}^{l}\right\|_{2}^{2}>\epsilon_{i j}^{y}\right] \leq P_{f_{2}} \\
& \mathbf{P}\left[\mathbf{g}_{i j}\left(\mathbf{Z}_{i j}\right)>\mathbf{0}\right] \leq \mathbf{P}_{f_{3}},
\end{array}
$$

where $E[\cdot]$ denotes expected value of a random variable, $P[\cdot]$ represents the probability of an event, and $P_{f_{i}}$, are scalars $(i=1,2)$ and vectors $(i=3)$ of assigned probabilities of failure, i.e., probabilities of violating constraints.

There are many approaches for solving stochastic programming problems. As a first step to apply the ATC methodology for the design of multilevel systems under uncertainty, we optimize with respect to the means of the random variables, and utilize an analytical first-order reliability method (FORM) to evaluate the reliability of satisfying the probabilistic constraints. Specifically, we adopt the hybrid mean value (HMV) algorithm to compute the most probable point (MPP) for each iterate of the optimization process [11]. It is emphasized that first-order methods yield exact results only if the limit-state (i.e., constraint) functions are linear, and random variables are normally distributed and uncorrelated. If these assumptions are violated, the obtained results are only approximate. These methods are used widely in literature due to their simplicity and efficiency despite their relative inaccuracy.

When solving a subproblem, the variance of random design variables is fixed. Nevertheless, ATC requires the iterative solution of the optimization subproblems. Therefore, the variances of the random design variables must be updated at each iteration of the ATC process before solving the optimization subproblems.

\section{PROPAGATION OF UNCERTAINTIES}

To update these stochastic quantities, we need to estimate the propagated uncertainties. This is achieved by linearizing the nonlinear function $\left\{\mathbf{R}_{i j}\right\}_{t}=$ $\left\{\mathbf{f}_{i j}\right\}_{t}\left(\mathbf{R}_{(i+1) k_{1}}, \ldots, \mathbf{R}_{(i+1) k_{c_{i j}}}, \mathbf{X}_{i j}, \mathbf{Y}_{i j}\right)=\left\{\mathbf{f}_{i j}\right\}_{t}(\mathbf{Z})$ that defines each entry $t$ of the random variable vector $\mathbf{R}_{i j}$ around the current iterate $\mu_{\mathbf{Z}}$ :

$$
\left\{\mathbf{f}_{i j}\right\}_{t}(\mathbf{Z}) \approx\left\{\mathbf{f}_{i j}\right\}_{t}\left(\mu_{\mathbf{Z}}\right)+\sum_{q=1}^{n} \frac{\partial\left\{\mathbf{f}_{i j}\right\}_{t}\left(\mu_{\mathbf{Z}}\right)}{\partial Z_{q}}\left(Z_{q}-\mu_{Z_{q}}\right),
$$

where $n$ is the dimensionality of the vector $\mathbf{Z}$. Assuming that all the random variables are statistically independent (uncorrelated), the first-order approximations of the mean and the variance of $\left\{\mathbf{R}_{i j}\right\}_{t}$ are given by

$$
E\left[\left\{\mathbf{R}_{i j}\right\}_{t}\right] \approx\left\{\mathbf{f}_{i j}\right\}_{t}\left(\mu_{\mathbf{Z}}\right)
$$


and

$$
\sigma_{\left\{\mathbf{R}_{i j}\right\}_{t}}^{2} \approx \sum_{q=1}^{n}\left(\frac{\partial\left\{\mathbf{f}_{i j}\right\}_{t}\left(\mu_{\mathbf{Z}}\right)}{\partial Z_{q}}\right)^{2} \sigma_{Z_{q}}^{2},
$$

respectively. The cascaded target and passed consistency parameter values are updated every time before solving problem $p_{i j}$ as follows: $\mathbf{r}_{i j}^{u}$ and $\mathbf{y}_{i j}^{u}$ are assigned the optimal values $\mu_{\mathbf{R}_{(i-1) m}}^{*}$ and $\mu_{\mathbf{Y}_{(i-1) m}}^{*}$, respectively, obtained by solving the parent problem $p_{(i-1) m}$; $\mathbf{y}_{(i+1) k_{1}}^{l}, \ldots, \mathbf{y}_{(i+1) k_{c_{i j}}}^{l}$ are assigned the optimal values $\mu_{\mathbf{Y}_{(i+1) k_{1}}}^{*} \ldots, \mu_{\mathbf{Y}_{(i+1) k_{c_{i j}}}^{*}}^{*}$ obtained by solving the children problems $p_{(i+1) k_{1}}, \ldots, p_{(i+1) k_{c_{i j}}} ; \mathbf{r}_{(i+1) k_{1}}^{l}, \ldots, \mathbf{r}_{(i+1) k_{c_{i j}}}^{l}$ are assigned the expected values of the responses as functions of the mean values obtained as optimal solutions of the children problems using Equation (5). Equation (6) is used to update the variance of the random design variables.

The linearization approach for modeling the uncertainty propagation is valid only in the neighborhood of the Taylor expansion point, i.e., the best current iterate. Therefore, large steps are not allowed during the optimization process. This condition is ensured by virtue of the consistency constraints in the ATC formulation.

\section{PISTON-RING/CYLINDER-LINER DESIGN CASE STUDY}

The piston-ring/cylinder-liner assembly houses the combustion process inside the engine. Regardless of the engine type (spark ignition or compression ignition), this assembly must provide a tightly sealed compartment during the various stages of the engine cycle, and must efficiently transmit the force of combustion to perform mechanical work. While a tight sealing is essential to reduce blow-by and prevent oil contamination, a certain degree of looseness is also required to permit the piston ring pack to slide along the liner surface. The main advantage of the separate liner is that it permits reconditioning of the worn surface. In the absence of a liner, the entire cylinder block would have to be replaced eventually.

Both rings and liners are generally comprised of tailored materials that are manufactured with controlled surface properties. While liners have traditionally been manufactured using cast iron, more advanced materials have recently been developed that provide better weight-tostrength ratio and high wear resistance under elevated temperature. Such materials include aluminum and metal matrix composites. Surface treatments such as electroplating have also been used to improve contact resistance between the piston ring and the cylinder liner.

After casting and machining to near net shape, the liner surface is both rough and plateau honed to impart a deliberate amount of roughness. For example, a fully manufactured cylinder liner will typically have a roughness profile such as that shown in Figure 3 . This roughness is considered critical for oil retention and lubrication. A comprehen- sive description of a liner or ring surface roughness profile requires several parameters including root mean square (RMS) of asperity height, asperity density, peak roughness, skewness, kurtosis, mean spacing core roughness, and many other parameters. Among these parameters, the surface RMS value is most commonly used as a single representative value to describe the surface condition. The surface RMS value $\left(R_{a}\right)$ is calculated by Equation (7),

$$
R_{a}=\sqrt{\frac{1}{n} \sum_{i=1}^{n}\left[s_{i}(p)-\overline{s(p)}\right]^{2}}
$$

where $s \underline{(p)}$ is the surface height at point $p$ in the surface profile, $\overline{s(p)}$ is the average height of the surface profile shown in Figure 3 , and $n$ is the number of sample points measured.

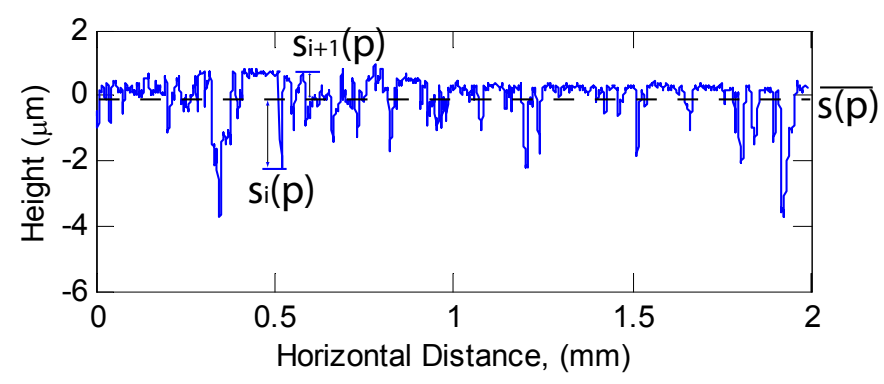

Figure 3: Typical Surface Roughness of Ring/Liner

MODEL DEVELOPMENT The flow of information necessary to predict the impact of piston-ring and cylinderliner surface roughness variations on brake-specific fuel consumption (BSFC) is shown in Figure 4. Surface rough-

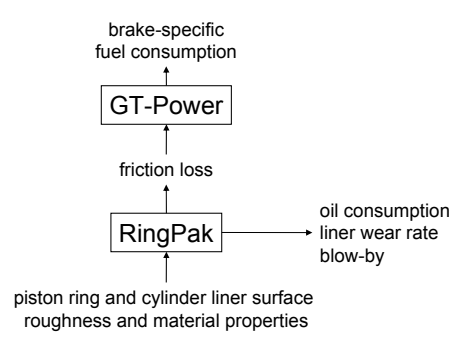

Figure 4: Piston ring / cylinder liner problem hierarchy

ness effects on friction loss are computed by the simulation package RingPak (Ricardo Inc., London, UK). The friction loss predictions are specific to a particular V6 gasoline engine with well known geometry, assembly characteristics, operating conditions, and material properties that were measured directly in the Automotive Laboratory of the University of Michigan [12]. The friction loss is then input to a simulation to predict BSFC, using the GT-Power software (Gamma Technology Inc., Westmond, IL).

Although the exact contribution of ring/liner friction losses 
to the total power loss of an engine depends on operating conditions and the characteristics of the specific engine under consideration, the piston assembly has been shown to typically account for $40-50 \%$ of total engine frictional losses [13]. The tribological characteristics of the piston-ring/liner interface alone have been shown to account for $20-30 \%$ of total frictional losses, and are directly related to a gamut of important engine performance metrics including power loss, fuel consumption, oil consumption, blow-by, and emissions [13]. In this investigation, it is assumed that $30 \%$ of the total friction loss is due to the tribological behavior of the ring/liner interface. Since the goal of this study is to understand how the manufacturing uncertainty propagates to the engine performance, the assumed power loss ratio is not of major consequence, as it does not impact the overall trends. However, it is known that the assumed power loss ratio has a significant impact on BSFC. Therefore more detailed measurements of the power loss ratio would be required, under multiple conditions, if the detailed output performance of a particular engine were at the center of the investigation [12].

Surrogate Modelling Due to the large computational requirements associated with the RingPak and GT- Power simulations, surrogate models were developed to predict the responses of the ring/liner subassembly and the engine(friction loss, oil consumption, blow-by, \& liner wear rate, and BSFC, respectively). Radial basis function artificial neural network models were trained, and the maximal error was computed at $5 \%$ using the leave-one-out crossvalidation technique.

PROBLEM FORMULATION Due to the simplicity of the given problem structure, we will use here a modified version of the notation introduced earlier: since there are only two levels with only one element in each, we skip element indices, and denote the upper-level element with subscript 0 and the lower-level element with subscript 1 . We use second indices to denote entries in the design variable vector of the lower-level element optimization problem. The design problem is to find optimal mean values $\mu_{X_{11}}$ and $\mu_{X_{12}}$ for the piston-ring and cylinder-liner surface roughness random variables $X_{11}$ and $X_{12}$, respectively, and optimal values for the deterministic design variables representing the material properties (Young's modulus $x_{13}$ and hardness $x_{14}$ ) of the liner that yield minimized expected value of brake-specific fuel consumption $R_{0}$ for a V6 gasoline engine. The optimal design is subject to constraints on liner wear rate, oil consumption, and blow-by. The critical link between the two commercial simulation models (RingPak and GT-Power) is the friction loss $R_{1}$ in the ring/liner assembly.

The top- and bottom-level ATC problems are formulated as

$$
\begin{array}{rc}
\min _{\mu_{R_{1}}, \epsilon^{r}} & \left(E\left[R_{0}\right]-T\right)^{2}+\epsilon^{r} \\
\text { subject to } & P\left[\left(\mu_{R_{1}}-r_{1}^{l}\right)^{2}>\epsilon^{r}\right] \leq P_{f_{1}}
\end{array}
$$

and

$$
\begin{array}{r}
\min _{\mu_{X_{11}}, \mu_{X_{12}}, x_{13}, x_{14}} \quad\left(E\left[R_{1}\right]-r_{1}^{u}\right)^{2} \\
\text { subject to } P\left[\text { liner wear rate }>2.4 \times 10^{-12} \mathrm{~m}^{3} / \mathrm{s}\right] \leq P_{f_{2}} \\
P\left[\text { blow-by }>4.25 \times 10^{-5} \mathrm{~kg} / \mathrm{s}\right] \leq P_{f_{3}} \\
\left.P \text { oil consumption }>15.3 \times 10^{-3} \mathrm{~kg} / \mathrm{hr}\right] \leq P_{f_{4}} \\
1.0 \mu \mathrm{m} \geq \mu_{X_{1}}, \mu_{X_{2}} \geq 0.1 \mu \mathrm{m} \\
340 \mathrm{GPa} \geq x_{3} \geq 50 \mathrm{GPa} \\
240 \mathrm{BHV} \geq x_{4} \geq 150 \mathrm{BHV},
\end{array}
$$

respectively, where $R_{1}=f_{1}\left(X_{11}, X_{12}, x_{13}, x_{14}\right)$ and $R_{0}=$ $f_{0}\left(R_{1}\right)$. The standard deviation of the surface roughnesses was assumed to be $0.1 \mu \mathrm{m}$, and remained constant throughout the ATC process. The assigned probability of failure $P_{f_{i}}$ was $0.13 \%$ for all four constraints, and the fuel consumption target $T$ was set to zero.

RESULTS The obtained results are summarized in Table 1. A Monte Carlo simulation was performed to assess

Table 1: Optimal ring/liner assembly design, expected fuel consumption and friction loss, and estimated variations

\begin{tabular}{|c|c|c|c|}
\hline & ATC results & Monte Carlo & Error \\
\hline \hline$E\left[R_{0}\right], k g / k W h$ & 0.2938 & 0.2882 & $1.9 \%$ \\
$\sigma_{R_{0}}, k g / k W h$ & 0.0083 & 0.0067 & $23.8 \%$ \\
\hline$E\left[R_{1}\right], k W$ & 0.2993 & 0.3205 & $6.6 \%$ \\
$\sigma_{R_{1}}, k W$ & 0.0608 & 0.0477 & $27.5 \%$ \\
\hline$\mu_{X_{11}}, \mu m$ & 0.6001 & - & - \\
$\mu_{X_{12}}, \mu m$ & 0.4264 & - & - \\
$x_{13}, G P a$ & 80 & - & - \\
$x_{14}, B H V$ & 220.84 & - & - \\
\hline
\end{tabular}

their accuracy. One million samples were generated using the mean and standard deviation values of the design variables. The linearization approach is more accurate for estimating mean values of responses; the error is larger when estimating standard deviations.

The evolution of the approximated expected values for the friction loss is depicted in Figure 5(a). It can be seen how the two problems "negotiate" on the value of the expected friction loss. Figure $5(\mathrm{~b})$ illustrates the propagation of uncertainty based on the obtained results.

\section{SUMMARY AND DISCUSSION}

A methodology for handling stochastic inputs in hierarchical multilevel system design optimization problems has been applied to the design of a piston-ring/cylinderliner engine subassembly. The methodology utilizes the probabilistic extension of the analytical target cascading methodology and a linearization technique to estimate the propagation of uncertainties. The reliability of satisfying the probabilistic constraints is calculated by means of the most probable point using the hybrid mean value algorithm. 


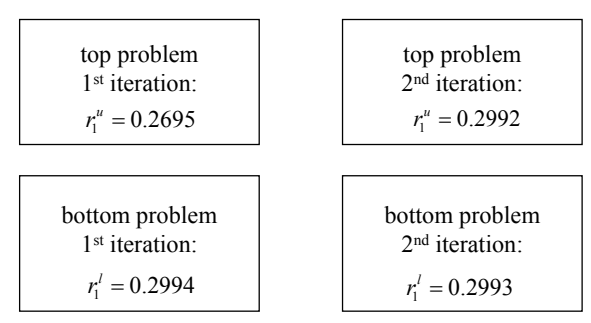

(a) Evolution of response values

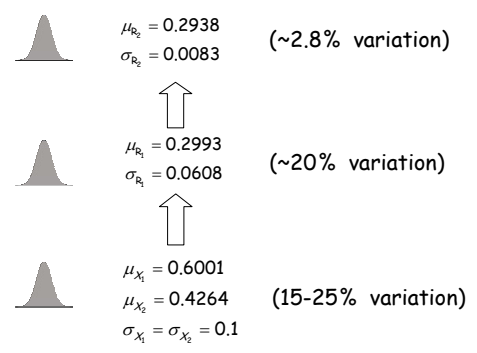

(b) Propagation of uncertainty

Figure 5: Illustration of results

The objective of the case study was to estimate the effects of variations in the cylinder/liner surface roughnesses on the brake-specific fuel consumption of the engine, as they propagate through two simulations. The case study demonstrated the potential and usefulness of the methodology for solving optimal design problems of multilevel systems under uncertainty. Nevertheless, the comparison of the results to the ones obtained by Monte Carlo simulation exposed the need of using more accurate techniques for solving the stochastic programming problems and propagating uncertainties. It is emphasized, however, that both of these issues can be addressed independently from the proposed ATC methodology for design of multilevel systems under uncertainty, which can therefore be characterized as viable.

\section{ACKNOWLEDGEMENTS}

This research was supported by research grants from the Dual Use Science and Technology Program of General Motors Company and the U.S. Army Tank-automotive and Armaments Command. This support is gratefully acknowledged.

\section{REFERENCES}

[1] H.M. Kim. Target Cascading in Optimal System Design. PhD thesis, University of Michigan, Ann Arbor, Michigan, 2001.

[2] H.M. Kim, N.F. Michelena, P.Y. Papalambros, and T. Jiang. "Target cascading in optimal system design". ASME Journal of Mechanical Design, 125(3):474-480, 2003.

[3] H.M. Kim, D.G. Rideout, P.Y. Papalambros, and J.L.
Stein. "Analytical target cascading in automotive vehicle design". ASME Journal of Mechanical Design, 125(3):481-489, 2003.

[4] N.F. Michelena, L. Louca, M. Kokkolaras, C.-C. Lin, D. Jung, Z. Filipi, D. Assanis, Papalambros P.Y., H. Peng, J. Stein, and M. Feury. "Design of an advanced heavy tactical truck: $A$ target cascading case study". SAE 2001 Transactions - Journal of Commercial Vehicles. Also appeared in the Proceedings of the 2001 SAE International Truck and Bus Meeting and Exhibition, Chicago, IL, Paper No. 2001-012793.

[5] H.M. Kim, M. Kokkolaras, L.S. Louca, G.J. Delagrammatikas, N.F. Michelena, Z.S. Filipi, P.Y. Papalambros, J.L. Stein, and D.N. Assanis. "Target cascading in vehicle redesign: A class VI truck study". International Journal of Vehicle Design, 29(3):1-27, 2002.

[6] M. Kokkolaras, R. Fellini, H.M. Kim, N.F. Michelena, and P.Y. Papalambros. "Extension of the target cascading formulation to the design of product families". Journal of Structural and Multidisciplinary Optimization, 24(4):293-301, 2002.

[7] L.S. Louca, M. Kokkolaras, G.J. Delagrammatikas, N.F. Michelena, Z.S. Filipi, P.Y. Papalambros, and D.N. Assanis. "Analytical target cascading for the design of an advanced technology heavy truck". In Proceedings of the 2002 ASME International Mechanical Engineering Congress and Exposition, New Orleans, LA. Paper No. IMECE-2002-32860.

[8] N.F. Michelena, H. Park, and P.Y. Papalambros. "Convergence properties of analytical target cascading". AlAA Journal, 41(5):897-905, May 2003.

[9] M. Kokkolaras and P.Y. Papalambros. "Analytical target cascading in design optimization of hierarchical multilevel systems under uncertainty". In 18th International Symposium on Mathematical Programming, (abstracts only), Copenhagen, Denmark, August 2003.

[10] Z. Mourelatos, D. Gorsich, M. Kokkolaras, and P.Y. Papalambros. "Modeling uncertainty in analytical target cascading". In SIAM Conference on Mathematics for Industry, (abstracts only), Toronto, Canada, October 2003.

[11] B.D. Youn, K.K. Choi, and Y.H. Park. "Hybrid analysis method for reliability-based design optimization". ASME Journal of Mechanical Design, 125(2):221232, 2003.

[12] Concurrent design of next-generation powertrains, manufacturing processes and materials. Technical report, The University of Michigan, Ann Arbor, MI, 2002.

[13] K.J. Patton, R.G. Nitschke, and J.B. Haywood. "Development and evaluation of a friction model for spark ignition engines", 1989. SAE paper no. 890836. 